\title{
Electron kinetics at the plasma interface
}

\author{
F. X. Bronold, H. Fehske, M. Pamperin, and E. Thiessen \\ Institut für Physik, Ernst-Moritz-Arndt-Universität Greifswald, D-17489 Greifswald, Germany
}

Received: date / Revised version: date

\begin{abstract}
The most fundamental response of an ionized gas to a macroscopic object is the formation of the plasma sheath. It is an electron depleted space charge region, adjacent to the object, which screens the object's negative charge arising from the accumulation of electrons from the plasma. The plasma sheath is thus the positively charged part of an electric double layer whose negatively charged part is inside the wall. In the course of the Transregional Collaborative Research Center SFB/TRR24 we investigated, from a microscopic point of view, the elementary charge transfer processes responsible for the electric double layer at a floating plasma-wall interface and made first steps towards a description of the negative part of the layer inside the wall. Below we review our work in a colloquial manner, describe possible extensions, and identify key issues which need to be resolved to make further progress in the understanding of the electron kinetics across plasma-wall interfaces.
\end{abstract}

PACS. 52.40.Hf Plasma-material interaction, boundary layer effects - 52.40.Kh Plasma sheaths - 68.49.Jk Electron scattering from surfaces - 68.49.Sf Ion scattering from surfaces (charge-transfer, sputtering, SIMS)

\section{Introduction}

The most fundamental manifestation of the interaction of a solid surface with an ionized gas is the formation of an electric double layer consisting, respectively, on the plasma and the solid side of the interface of an electron-depleted and an electron-rich space charge region. It arises because electrons are deposited more efficiently inside or on top of the solid, depending on the electronic structure, than they are extracted from it by the neutralization of ions and the de-excitation of heavy neutral particles. That an electric double layer forms at a plasma-facing solid (plasma interface), having a negative part inside the solid and a positive part inside the plasma, is known since the beginnings of gaseous electronics [1. A microscopic understanding of the charge transfer across the plasma interface on par with an understanding of charge transfer between two gaseous plasmas [2, 3, 4, 5] or between two solids, such as, Schottky contacts [6] is however still lacking. What has been studied in depth so far is only the merging of the plasma-based part of the double layer-the plasma sheath-with the quasineutral bulk plasma [7,8,9] and how it is affected by the emissive properties of the surface, that is, by electron/ion reflection and electron emission [10,11,12.

As far as the theoretical description of this fundamental electronic response of the plasma interface is concerned it is usually assumed that the processes inside the solid occur on spatial/temporal scales too small/fast to affect the plasma [13]. For the plasma species the solid is thus assumed to be only a reservoir characterized by surface parameters, such as, absorption, reflection, and emission probabilities, which have to be obtained either by quantum-mechanical calculations or by separate measurements. Encapsulating the physics of charge transfer across the plasma interface into a set of surface parameters, that is, considering electron absorption, reflection, and extraction as elementary surface collision processes has a long tradition in plasma physics (see [14, 15, 16, 17, 18, 19 for representative recent applications of this philosophy). Usually the surface parameters are assumed to be just numbers, independent of energy and angle, which in general is of course not true. The crude description reflects simply the fact that in most cases the surface parameters have been neither worked out theoretically nor measured experimentally. In particular at low impact energies the data base is rather sparse or outdated as emphasized for the particular case of secondary electron emission by Tolias 20. It is only recently that experimental work started anew to determine at energies relevant for low-temperature plasma applications surface parameters, such as, the electron backscattering probability [21] or the secondary electron emission coefficient [22, 23].

Characterizing charge transfer across the plasma interface by a set of (energy- and angle-dependent) surface parameters is justified as long as the scales of charge transport/relaxation inside the plasma and inside the solid are well separated. In situations, however, where they approach each other, as we expect it to occur soon in arrays of integrated microdischarges [24,25], because of the continuing miniaturization efforts in this field [26], it is no longer sufficient to treat electron deposition and extraction across the plasma interface as elementary surface collision processes. Instead it will be necessary to describe the 
charge transport across the interface selfconsistently with the charge dynamics on both sides of it. Such an approach is also required if one wants to understand in detail how the electronic non-equilibrium of the plasma is transferred to the solid, or if the physical system of interest consists of a plasma and a solid component, as it is the case for the plasma bipolar junction transistor [27.

Motivated primarily by the prospects of solid-based opto-electronic plasma devices [24, 25, 26, 27, but also with an eye on dielectric barrier discharges $28,29,30$, we initiated in the framework of the Transregional Collaborative Research Center SFB/TRR24 a still on-going effort to understand the charge transport, that is, the electron kinetics across the plasma interface from a microscopic point of view. Part of our work is devoted to the calculation of surface parameters (which may be also functions of energy and angle). Besides assisting future experimental efforts to measure them the parameters will help to make the modeling of the plasma-wall interaction more realistic. Considering electron deposition and extraction as elementary surface collision processes we calculated for dielectric surfaces probabilities for electron sticking/backscattering [31. 32, 33, 34, 35 and secondary electron emission 36, 37 and investigated how electronic correlations affect the neutralization of ions on metallic surfaces 38,39 . In addition we started to explore the fate of the surplus electrons inside the wall, that is, the solid-based negative part of the electric double layer 40,41 and proposed a method to measure the charge of a dielectric particle embedded in a plasma optically by Mie scattering [42, 43, 44]. In the following sections we colloquially review our work, present numerical results for material systems not considered in our previous work, or of exploratory calculations extending it, and identify key issues which need to be resolved for making further progress in the microscopic understanding of charge transfer across plasma-wall interfaces.

\section{Electron absorption and backscattering}

The interaction of electrons with surfaces is central for a great variety of surface diagnostics as well as materials processing techniques. It has been studied in great detail (see references in [31,32]). This knowledge however is not of immediate use for the modeling of electron-surface interaction in plasma applications, such as, dielectric barrier discharges, Hall thrusters, or the divertor region of fusion plasmas. The reason is the difference in the electron energies. Whereas in surface diagnostics, for instance, in electron microscopy, the energy of the electron probing the surface is at least $100 \mathrm{eV}$ the energy of electrons hitting the wall of a bounded plasma is typically less than $10 \mathrm{eV}$. In this energy range little is known quantitatively about the interaction of electrons with surfaces. The probability with which a low energy electron gets stuck in the surface and hence contributes to the solid-based negative part of the electric double layer is essentially unknown for the materials used as plasma walls. Very often it is thus assumed the probability is unity (perfect absorber assumption), irrespective of the angle of incidence and the wall material.
An electron, however, impinging on a solid surface is either reflected, inelastically scattered or temporarily deposited to the surface with possible trapping states (or sites) depending on the electron affinity of the surface. The perfect absorber model can thus not be universally valid.

To overcome the limitations of the perfect absorber model we initially modelled electron trapping/sticking at low energies as a physisorption process in the surface's image potential using rate equations for the occupancies of the image states $33,34,35$, and relying on methods developed for describing the adsorption of neutral atoms [45, 46]. This approach, applicable to electro-negative dielectrics, such as LiF, yields very small sticking probabilities. Due to lack of experimental data it is presently unclear how realistic these results are. We also investigated electron absorption by electro-positive dielectrics [31,32, such as, $\mathrm{Al}_{2} \mathrm{O}_{3}$ or $\mathrm{SiO}_{2}$. Since they are more commonly used in plasma applications we discuss the approach applied to them in more detail.

An electron hitting a dielectric with positive electron affinity may enter, after a successful transmission through the surface potential, the conduction band of the dielectric. Inelastic scattering due to phonons may then push the electron back to the interface and, after a successful transmission through the surface potential in the reverse direction, back to the plasma. In general, the probability for this chain of events is finite. Hence, the sticking probability should be less than unity with the particular numerical value depending on the electronic structure of the surface and the efficiency of the scattering processes.

Utilizing the electron's large penetration depth at the energies of a few electron volts [47, typical for plasma applications, we showed that the chain of events described in the previous paragraph gives rise to a sticking probability $S(E, \xi)$ which is the product of the probability $\mathcal{T}(E, \xi)$ for quantum-mechanical transmission through the surface potential and the probability to stay inside the surface despite of inelastic backscattering inside it [31,32]. To make the connection between absorption by and backscattering from the dielectric surface explicit, we recast the expression for $S(E, \xi)$ in the form

$$
S(E, \xi)=1-\int_{\chi}^{E} d E^{\prime} \int_{0}^{1} d \xi^{\prime} R\left(E \xi \mid E^{\prime} \xi^{\prime}\right),
$$

where $R\left(E \xi \mid E^{\prime} \xi^{\prime}\right)$ encodes the backscattering of an electron hitting the surface with energy $E$ and direction cosine $\xi$ to a state with energy $E^{\prime}$ and direction cosine $\xi^{\prime}$ (see Figure 1 for the definition of the cosines and energies). It consists of two parts,

$$
\begin{aligned}
R\left(E \xi \mid E^{\prime} \xi^{\prime}\right) & =R(E, \xi) \delta\left(E-E^{\prime}\right) \delta\left(\xi-\xi^{\prime}\right) \\
& +\delta R\left(E \xi \mid E^{\prime} \xi^{\prime}\right),
\end{aligned}
$$

with $R(E, \xi)=1-\mathcal{T}(E, \xi)$ the probability for quantummechanical reflection by the surface potential and

$$
\begin{aligned}
\delta R\left(E \xi \mid E^{\prime} \xi^{\prime}\right) & =\frac{\partial \eta^{\prime}}{\partial \xi^{\prime}} \mathcal{T}(E, \xi) \rho\left(E^{\prime}\right) \mathcal{B}\left(E \eta \mid E^{\prime} \eta^{\prime}\right) \mathcal{T}\left(E^{\prime} \xi^{\prime}\right) \\
& \times \theta\left(\xi^{\prime}-\sqrt{1-\bar{m}_{e}}\right)
\end{aligned}
$$


with

$$
\mathcal{B}\left(E \eta \mid E^{\prime} \eta^{\prime}\right)=\frac{Q\left(E \eta \mid E^{\prime} \eta^{\prime}\right)}{\int_{0}^{1} d \eta^{\prime} \int_{0}^{E} d E^{\prime} \rho\left(E^{\prime}\right) Q\left(E \eta \mid E^{\prime} \eta^{\prime}\right)}
$$

the probability for diffuse backscattering. The differential coefficient $\partial \eta^{\prime} / \partial \xi^{\prime}$ arises because the direction cosines inside $\left(\eta\right.$ and $\left.\eta^{\prime}\right)$ and outside $\left(\xi\right.$ and $\left.\xi^{\prime}\right)$ the surface are different because of the mismatch between the electron masses and the three-dimensional potential step mimicking the surface potential. Due to conservation of the total energy $E$ and the lateral momentum $\boldsymbol{K}$ they are connected by

$$
1-\eta^{2}=\frac{E-\chi}{\bar{m}_{e} E}\left(1-\xi^{2}\right)
$$

with $\bar{m}_{e}=m_{*} / m_{e}$ the mass of a conduction band electron in units of the bare electron mass and $\chi>0$ the electron affinity of the dielectric. Using this relation for the postcollision direction cosines yields the relation $\partial \eta^{\prime} / \partial \xi^{\prime}$.

For the model shown in Figure 1 the transmission probability is given by

$$
\mathcal{T}(E, \xi)=\frac{4 \bar{m}_{e} k p}{\left(\bar{m}_{e} k+p\right)^{2}}
$$

with $k=\sqrt{E-\chi} \xi$ and $p=\sqrt{\bar{m}_{e} E} \eta$ the $z$-components of the electron momenta outside and inside the wall. In all formulae atomic units are used, that is, energy is measured in Rydbergs and lengths in Bohr radii. Since the signs of $k$ and $p$ are always the same in (6) we define the direction cosines $\xi$ and $\eta$ referenced, respectively, to the electron momenta outside and inside the wall, by their absolute values: $\xi=|\cos \beta|$ and $\eta=|\cos \theta|$. This choice simplifies also the calculation of the function $Q\left(E \eta \mid E^{\prime} \eta^{\prime}\right)$ which is the essential part of $\delta R\left(E \xi \mid E^{\prime} \xi^{\prime}\right)$, the function describing diffuse backscattering by the surface.

The function $Q\left(E \eta \mid E^{\prime} \eta^{\prime}\right)$ is proportional to the probability for an electron penetrating the surface in a state with (total) energy $E$ and direction cosine $\eta$ to backscatter after an arbitrary number of internal scattering events towards the interface in a state with energy $E^{\prime}$ and direction cosine $\eta^{\prime}$. It is determined by a recursion relation derived from the principle of invariant embedding [48, 49, 50. The invariant embedding principle is particularly well suited for our purpose because it enables us to perform an expansion in the number of backscattering events which converges very fast because backscattering is usually much less likely than forward scattering. In fact, we can truncate the expansion already after one backscattering event. What is an advantage for the invariant embedding approach is a serious drawback for Monte Carlo simulations. Sampling rarely occurring backscattering trajectories is computationally demanding. Standard Monte Carlo techniques are thus not particularly well-suited for the investigation of electron backscattering.

In 31,32 we derived the recursion relation for the function $Q\left(E \eta \mid E^{\prime} \eta^{\prime}\right)$ under the assumption that the dielectric surface is at room temperature and the impinging electron has initially an energy $E<E_{g}$, where $E_{g}$ is the band gap of the dielectric. The first assumption implies that the scattering occurs primarily due to emission of optical phonons while the second assumption guarantees that Coulomb-driven electron energy dissipation can be ignored. Taking then into account that a forward scattering event leads in a very good approximation only to an energy loss $\omega$, where $\omega$ is the energy of the phonon, leaving the propagation direction of the electron however unchanged, $Q\left(E \eta \mid E^{\prime} \eta^{\prime}\right)$ can be written as

$$
Q\left(E \eta \mid E^{\prime} \eta^{\prime}\right)=\sum_{m=0}^{M_{\text {open }}} Q_{m}^{1}\left(E ; \eta \mid \eta^{\prime}\right) \delta\left(E-E^{\prime}-\omega_{m}^{1}\right)
$$

with $M_{\text {open }}$ the number of forward scattering events at most possible for the initial energy $E, \xi$ the initial direction cosine, and $\omega_{m}^{1}=(1+m) \omega$. The expansion coefficients $Q_{m}^{1}\left(E ; \eta \mid \eta^{\prime}\right)$ satisfy a linear recursion relation, to be found in [31 32], which can be solved numerically quite efficiently for given values of $E, \eta$, and $\eta^{\prime}$.

The physical picture behind our approach of calculating the electron sticking and backscattering probabilities for a dielectric surface with positive electron affinity is illustrated in Figure 1 together with representative results for an $\mathrm{Al}_{2} \mathrm{O}_{3}$ surface. First let us consider the illustration on the left. It shows that for a dielectric with positive electron affinity diffuse backscattering from the surface is due to an internal multi-phonon cascade consisting of a finite number of electron trajectories characterized by a single backscattering and an increasing number of forward scattering events. Each trajectory represents a backscattering channel contributing to the diffuse backscattering from the surface if the electron moving along such a trajectory traverses the surface potential successfully from the solid to the plasma side.

Let us now turn to the numerical data shown in the middle and the right panel of Figure 1. The middle panel gives $S(E, \xi)$ over the whole range of direction cosines $\xi$ and energies up to $9 \mathrm{eV}$ above which Coulomb-driven scattering processes should be taken into account. In principle this is possible. Preliminary work indicated that the recursion relations, containing in this case also energy integrals, can still be solved efficiently by numerical means. Work in this direction is in progress but will not be reported here. The sticking probability $S(E, \xi)$ in the white region of the $(E, \xi)$-plane is identically zero. It is the region of total reflection at the three-dimensional potential step characterizing the surface potential. Below the yellow line, given by $\xi=\sqrt{1-\bar{m}_{e}}$, the sticking probability is given by the quantum-mechanical transmission probability, that is, $S(E, \xi)=\mathcal{T}(E, \xi)$. This is a consequence of the mass mismatch $\bar{m}_{e}=m_{*} / m_{e}<1$ and the conservation of total energy and lateral momentum which force the perpendicular energy of an electron impinging on the surface with a direction cosine $\xi<\sqrt{1-\bar{m}_{e}}$ to be less than $\chi$, the depth of the surface potential, once it crossed the interface from the plasma side. Since at room temperature the electron cannot gain energy by absorbing phonons it is thus immediately confined to the surface once it entered it. Only above the yellow line, that is, for $\xi>\sqrt{1-\bar{m}_{e}}$ phonon 

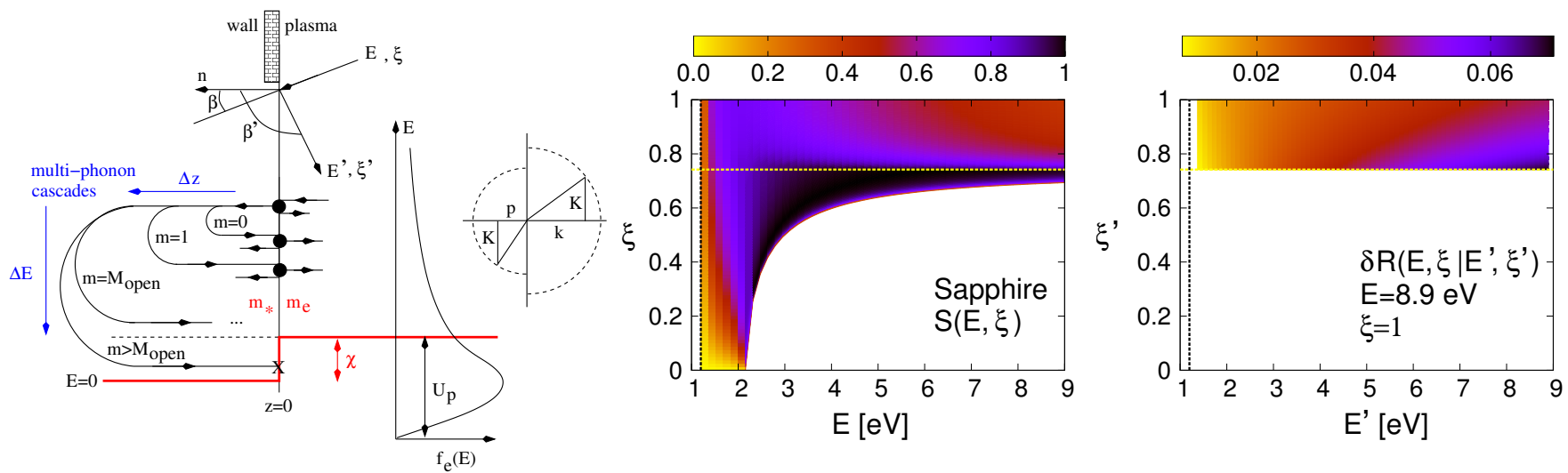

Fig. 1. (Color online) Left panel: Interface model and principal idea of the calculational approach used for studying electron absorption/backscattering by dielectric walls with positive electron affinity $\chi>0$. The direction cosines inside $(\eta)$ and outside $(\xi)$ the surface can be deduced from the two semicircles. Central panel: Angle-resolved electron sticking probability $S(E, \xi)$ for $\mathrm{Al}_{2} \mathrm{O}_{3}$ (sapphire) with material parameters $m_{e} / m_{*}, \chi, E_{g}$, and $\varepsilon$ as listed in Table 1 . Below the yellow dashed line, indicating $\xi=\sqrt{1-\bar{m}_{e}}$, inelastic backscattering has no effect on the sticking probability. In the white region $S(E, \xi)=0$ due to total reflection of the electron by the potential step mimicking the surface potential. Right panel: The function $\delta R\left(E \xi \mid E^{\prime} \xi^{\prime}\right)$, describing diffuse backscattering of an electron with energy $E$ and direction cosine $\xi$ into a state with energy $E^{\prime}$ and direction cosine $\xi^{\prime}$, for an electron hitting the $\mathrm{Al}_{2} \mathrm{O}_{3}$ surface with $E=8.9 \mathrm{eV}$ and $\xi=1$. Below the dotted yellow line $\delta R\left(E \xi \mid E^{\prime} \xi^{\prime}\right)=0$ because diffuse backscattering cannot lead to post-collision cosines $\xi^{\prime}<\sqrt{1-\bar{m}_{e}}$

emission may bring the electron back to the interface. The possibility of crossing it also from the solid side leads then in this part of the $(E, \xi)$-plane to $S(E, \xi) \leq \mathcal{T}(E, \xi)$.

Having a numerical value for the sticking probability $S(E, \xi)$ is enough for determining for instance the charge of a dust particle in a plasma. If one is however interested in the feedback the surface has to the plasma the electron backscattering probability $R\left(E \xi \mid E^{\prime} \xi^{\prime}\right)$ is the more important quantity. It consists of the quantum-mechanical (specular) backscattering probability $R(E, \xi)$ and the diffuse backscattering probability $\delta R\left(E \xi \mid E^{\prime} \xi^{\prime}\right)$. The latter is shown in the right panel of Figure 1 for an electron hitting an $\mathrm{Al}_{2} \mathrm{O}_{3}$ surface with $E=8.9 \mathrm{eV}$ and $\xi=1$. Similar plots arise for other values of $E$ and $\xi$. Below the yellow line, given now by $\xi^{\prime}=\sqrt{1-\bar{m}_{e}}$, diffuse backscattering is not possible because the mass mismatch in conjunction with the conservation of total energy and lateral momentum prevent post-collision cosines $\xi^{\prime}<\sqrt{1-\bar{m}_{e}}$. Diffuse backscattering in these directions is thus impossible.

The data presented in Figure 1 are obtained for a laterally homogeneous surface. Only then the lateral momentum is conserved leading to total reflection. In reality, surfaces in contact with a plasma are for sure laterally inhomogeneous. Lateral momentum is thus not conserved and total reflection suppressed. In [31,32] we also considered this case, based on a simple model for interfacial scattering 51] which destroys the lateral homogeneity. We then found astonishingly good agreement between calculated values for $S(E, \xi=1)$ and data obtained from electronbeam scattering experiments on $\mathrm{MgO} 52$ and $\mathrm{SiO}_{2}[53$ surfaces indicating that our approach captures the essential processes responsible for low-energy electron backscattering from electro-positive dielectrics.

So far we focused on electron energy relaxation due to scattering by optical phonons. But other scattering pro-

\begin{tabular}{|l|lllllll|}
\hline & $\frac{m_{e}}{m_{h}}$ & $\frac{m_{e}}{m_{*}}$ & $\begin{array}{l}k_{B} T_{*} \\
(\mathrm{eV})\end{array}$ & $\begin{array}{l}k_{B} T_{h} \\
(\mathrm{eV})\end{array}$ & $\begin{array}{l}\chi \\
(\mathrm{eV})\end{array}$ & $\begin{array}{l}E_{g} \\
(\mathrm{eV})\end{array}$ & $\varepsilon$ \\
\hline $\mathrm{SiO}_{2}$ & 1.0 & 1.3 & 0.2 & 0.2 & 1.2 & 9.0 & 4 \\
$\mathrm{Al}_{2} \mathrm{O}_{3}$ & 1.0 & 2.2 & 0.2 & 0.2 & 1.2 & 8.7 & 9.9 \\
$\mathrm{MgO}$ & 1.0 & 2.5 & 0.2 & 0.2 & 1.2 & 7.8 & 9.8 \\
\hline \hline & $\frac{m_{e}}{m_{i}}$ & & $k_{B} T_{e}$ & $k_{B} T_{i}$ & $I$ & $\Gamma$ & \\
& $\left(10^{-4}\right)$ & & $(\mathrm{eV})$ & $(\mathrm{eV})$ & $(\mathrm{eV})$ & $(\mathrm{eV})$ & \\
\hline $\mathrm{H}^{+} e$ & 5.4 & & 2.0 & 0.2 & 13.6 & 2.0 & \\
\hline
\end{tabular}

Table 1. Material parameters used in the calculations for the perfectly absorbing collisionless plasma-wall interface. The image shift of the ion's ionization level $I$ is neglected to keep the model as simple as possible. It should be also noticed that the parameters give only an orientation. For real surfaces used in actual experiments the values may deviate from the ones given due to materials science aspects not addressed in this work.

cesses can be included as well. The numerical effort increases, in particular, if Coulomb-driven scattering processes are included, such as, electron-hole pair generation due to the impacting electron, becoming important for $E>E_{g}$. Still, we expect the approach to remain more efficient than Monte Carlo simulations. It should thus be a rather useful tool for analyzing the recent experimental data obtained for electron backscattering from plasma walls 21 and for generating for various materials data for $R\left(E \xi \mid E^{\prime} \xi^{\prime}\right)$ and $S(E, \xi)$ to be used in plasma modeling.

\section{Electron extraction}

Charge extraction by the neutralization of ions and/or the de-excitation of neutral particles is an important elementary surface collision process because it may provide secondary electrons which in turn affect the overall charge 
balance of the plasma. At high impact energies electrons are ejected from the surface due to the transfer of the kinetic energy associated with the center-of-mass motion of the projectile to the surface (kinetic electron ejection) whereas at low impact energies electrons are released due to the transfer of the energy stored in the internal motion of the projectile's constituents (potential electron ejection) [54. In the SFB/TRR24 we were particularly interested in a theoretical description of potential electron ejection from plasma walls. Besides the well-known twoelectron Auger processes (Ref. 55 and below) we investigated secondary electron emission due to auto-detaching negative ions [36, 37] and analyzed resonant neutralization of ions with an eye on mixed-valence and Kondo resonances 38, 39.

Our work on electron extraction from surfaces due to impacting atomic particles is based on multi-channel Anderson-Newns models [56] parameterized by experimental energies and target-projectile interactions deduced from considerations based on image charges [57,58. The models act on the internal states of the projectiles taking their classical center-of-mass motion in the surface potential into account by time-dependent matrix elements. Recasting the models in terms of pseudo-particles [59, representing the projectile's electronic configurations, as proposed in a pioneering work by Langreth and Nordlander 60, quantum kinetic equations for their occurrence probabilities can be derived and numerically solved $60,61,62$.

For the de-excitation of metastable $\mathrm{N}_{2}^{*}$ molecules on dielectric surfaces we obtained good agreement with experimental data 36,37. The secondary electron emission coefficients we find for the neutralization of $\mathrm{He}^{+}$on metal surfaces are also of the correct order of magnitude indicating that the semiempirical approach is working. From our point of view, the semiempirical approach is also the most suitable for describing elementary surface collision processes at plasma walls which are in most cases not well enough characterized to justify a more elaborate theoretical description.

Although our prime motivation stems from the desire to characterize elementary surface collision processes at plasma walls we investigated also the neutralization of strontium ions on gold surfaces [38,39, because experiments 63,64 indicated that it is perhaps driven by a mixed-valence resonance and thus by a feature which is paradigmatic for correlated quantum impurity systems. Our theoretical analysis could not fully support this conjecture but we found evidence for it deserving further exploration. The $\mathrm{Sr}^{+}: \mathrm{Au}$ system is not relevant of course for plasma applications. It shows however that the charge transfer processes taking place at plasma interfaces can be embedded in a context making them interesting to a larger group of physicists.

To give a better idea of how electron ejection at low impact energies can be approached by Anderson-Newns models we discuss secondary electron emission due to the neutralization of a $\mathrm{He}^{+}$ion on a tungsten surface in more detail. For the purpose of this paper, which is to review our work performed in the SFB/TRR24, the discussion (a)

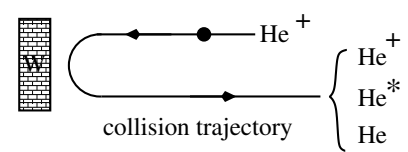

(c)

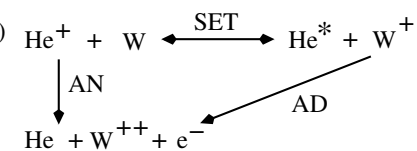

(d) $\varepsilon_{1}$

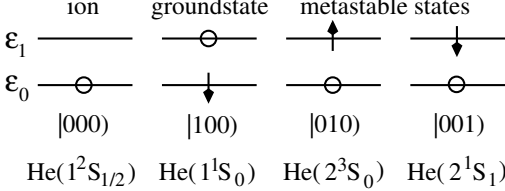

(b)

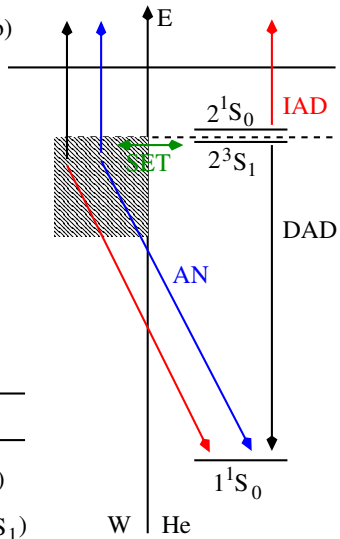

Fig. 2. (Color online) Schematic representation of an Anderson-Newns model based analysis of the neutralization of a helium ion on a tungsten surface characterized by a Fermi energy $E_{\mathrm{F}}=6.4 \mathrm{eV}$ and a work function $\Phi=4.5 \mathrm{eV}$. Other projectile-target combinations and other collision scenarios can be treated in the same spirit. Panel (a) shows a collision trajectory leading to time-dependent matrix elements and thus to the necessity to use quantum-kinetic equations for the theoretical analysis of the neutralization process. The reaction channels included into our modeling are indicated in the (on scale) energy (b) and the channel (c) diagram. In the energy diagram the image shifts are neglected for simplicity. The energy levels given for the projectile are the ones far away from the surface. The helium ion may capture an electron from the metal by a single-electron transfer (SET), changing its configuration from $\mathrm{He}^{+}\left(1 \mathrm{~s} ; 1^{2} \mathrm{~S}_{1 / 2}\right)$ to either $\mathrm{He}^{*}\left(1 \mathrm{~s} 2 \mathrm{~s} ; 2^{3} \mathrm{~S}_{1}\right)$ or $\mathrm{He}^{*}\left(1 \mathrm{~s} 2 \mathrm{~s} ; 2^{1} \mathrm{~S}_{0}\right)$, and subsequently Auger de-excite (AD) to $\mathrm{He}\left(1 \mathrm{~s}^{2} ; 1^{1} \mathrm{~S}_{0}\right)$ releasing thereby a secondary electron. The $\mathrm{He}^{+}\left(1 \mathrm{~s} ; 1^{2} \mathrm{~S}_{1 / 2}\right)$ ion may however also Auger neutralize (AN) to $\mathrm{He}\left(1 \mathrm{~s}^{2} ; 1^{1} \mathrm{~S}_{0}\right)$ which also leads to the ejection of an electron. Panel (d) depicts the twolevel system to which the electronic configurations of the projectile involved in the collision process give rise to with $\varepsilon_{0}$ the first ionization energy of helium $(24.6 \mathrm{eV})$ and $\varepsilon_{1}$, depending on the spin orientation, the ionization energy of the singlet $(4 \mathrm{eV})$ or triplet $(4.8 \mathrm{eV})$ metastable state.

will stay at the qualitative level, mathematical details will be given elsewhere 65 .

The essence of our approach is illustrated in Figure 2 Panel (a) of this figure shows a collision trajectory. For simplicity we assume the $\mathrm{He}^{+}$ion to hit the surface perpendicularly. Hence, the position of the projectile in front of the surface is given by $z(t)=z_{\mathrm{tp}}+v|t|$, where $z_{\mathrm{tp}}$ is the turning point, $v$ is the velocity of the projectile and $t$ runs from $-t_{\max }$ to $t_{\max }$ with $t_{\max }$ large enough to decouple the projectile from the target. Other collision angles can be also treated. At the level of our models it just leads to a velocity-induced smearing out of the target's Fermi edge 66. Below we present in fact data for grazing incident. Two main neutralization routes, put together in panels (b) and (c), are operative, each one leading to the ejection of an electron. The ion can either Auger neutralize $(\mathrm{AN})$ to the groundstate of the atom or Auger de-excite $(\mathrm{AD})$ to it in case a metastable configuration is formed before via a single-electron transfer. In that case a di- 
rect (DAD) and an indirect (IAD) channel exist. Assuming without loss of generality the electron of the $\mathrm{He}^{+}$ion to have spin up, the electronic configurations involved in the neutralization processes summarized in panel (c) are $\mathrm{He}^{+}\left(1^{2} \mathrm{~S}_{1 / 2}\right)$ for the ion, $\mathrm{He}\left(1^{1} \mathrm{~S}_{0}\right)$ for the groundstate, $\mathrm{He}^{*}\left(2^{3} \mathrm{~S}_{1}\right)$ for the triplet metastable state, and $\mathrm{He}^{*}\left(2^{1} \mathrm{~S}_{0}\right)$ for the singlet metastable state. They give rise to the twolevel system shown in panel $(d)$.

Instead of using Fermi operators acting on the twolevel system we prefer to represent the electronic configurations of the projectile by pseudo-operators. Recalling that the $1 \mathrm{~s}$ and $2 \mathrm{~s}$ shell of helium are involved in building up the electronic states of the projectile, we introduce operators $e^{\dagger}, s_{1 \downarrow}^{\dagger}, s_{2 \uparrow}^{\dagger}$, and $s_{2 \downarrow}^{\dagger}$ by defining

$$
\begin{aligned}
& |000\rangle=e^{\dagger}|\mathrm{vac}\rangle, \quad|100\rangle=s_{1 \downarrow}^{\dagger}|\mathrm{vac}\rangle, \\
& |010\rangle=s_{2 \uparrow}^{\dagger}|\mathrm{vac}\rangle, \quad|001\rangle=s_{2 \downarrow}^{\dagger}|\mathrm{vac}\rangle .
\end{aligned}
$$

They denote, respectively, the ion $\mathrm{He}^{+}\left(1^{2} \mathrm{~S}_{1 / 2}\right)$, the groundstate atom $\mathrm{He}\left({ }^{1} \mathrm{~S}_{0}\right)$, and the triplet and singlet metastable states, $\mathrm{He}^{*}\left(2^{3} \mathrm{~S}_{1}\right)$ and $\mathrm{He}^{*}\left(2^{1} \mathrm{~S}_{0}\right)$. Employing the reasoning of our previous work 36,38 , the Hamiltonian describing the neutralization of a $\mathrm{He}^{+}\left(1^{2} \mathrm{~S}_{1 / 2}\right)$ ion on a tungsten surface within the scenario summarized in Figure 2 becomes

$$
\begin{aligned}
H(t) & =\varepsilon_{s}^{*}(t) s_{2 \downarrow}^{\dagger} s_{2 \downarrow}+\varepsilon_{t}^{*}(t) s_{2 \uparrow}^{\dagger} s_{2 \uparrow}+\varepsilon_{g}(t) s_{1 \downarrow}^{\dagger} s_{1 \downarrow} \\
& +\sum_{\boldsymbol{q} \sigma} \varepsilon_{\boldsymbol{q} \sigma}(t) c_{\boldsymbol{q} \sigma}^{\dagger} c_{\boldsymbol{q} \sigma}+\sum_{\boldsymbol{k} \sigma} \varepsilon_{\boldsymbol{k} \sigma} c_{\boldsymbol{k} \sigma}^{\dagger} c_{\boldsymbol{k} \sigma} \\
& +\sum_{\boldsymbol{k} \sigma}\left[V_{\boldsymbol{k} \sigma}^{\mathrm{SET}}(t) c_{\boldsymbol{k} \sigma}^{\dagger} e^{\dagger} s_{2 \sigma}+\text { H.c. }\right] \\
& +\sum_{\boldsymbol{k}_{1} \boldsymbol{k}_{2} \boldsymbol{k}^{\prime} \sigma}\left[V_{\boldsymbol{k}_{1} \boldsymbol{k}_{2} \boldsymbol{k}^{\prime} \sigma \downarrow}^{\mathrm{AN}}(t) c_{\boldsymbol{k}^{\prime} \sigma}^{\dagger} s_{1 \downarrow}^{\dagger} e c_{\boldsymbol{k}_{1} \downarrow} c_{\boldsymbol{k}_{2} \sigma}+\text { H.c. }\right] \\
& +\sum_{\boldsymbol{k} \boldsymbol{k}^{\prime} \sigma}\left[V_{\boldsymbol{k} \boldsymbol{k}^{\prime} \sigma}^{\mathrm{DAD}}(t) c_{\boldsymbol{k}^{\prime} \sigma}^{\dagger} c_{\boldsymbol{k} \sigma} s_{1 \downarrow}^{\dagger} s_{2 \downarrow}+\text { H.c. }\right] \\
& +\sum_{\boldsymbol{k q} \sigma}\left[V_{\boldsymbol{k} \boldsymbol{q} \sigma}^{\mathrm{IAD}}(t) c_{\boldsymbol{q} \sigma}^{\dagger} c_{\boldsymbol{k} \downarrow} s_{1 \downarrow}^{\dagger} s_{2 \sigma}+\text { H.c. }\right]
\end{aligned}
$$

where the operator $c_{\boldsymbol{k} \sigma}^{\dagger}$ creates an electron with momentum $\boldsymbol{k}$ and spin $\sigma$ in the conduction band of the metal and the operator $c_{\boldsymbol{q} \sigma}^{\dagger}$ puts an electron in an unbound projectile state with momentum $\boldsymbol{q}$ and spin $\sigma$. In terms of pseudo-particle operators the physical meaning of the various terms of the Hamiltonian is very transparent. For instance, the fourth line of 10 denotes Auger neutralization, where the projectile transfers from the ion configuration to the groundstate configuration while creating an electron in state $\left|\boldsymbol{k}^{\prime} \sigma\right\rangle$ and destroying two metal electrons, one in state $\left|\boldsymbol{k}_{1} \downarrow\right\rangle$ and one in state $\left|\boldsymbol{k}_{2} \sigma\right\rangle$.

The energies $\varepsilon_{g}(t), \varepsilon_{s}^{*}(t), \varepsilon_{t}^{*}(t)$, and $\varepsilon_{\boldsymbol{q} \sigma}(t)$ of the projectile's electronic configurations are time-dependent because of polarization effects which depend on the projectile's distance to the surface and thus its instantaneous position in front of the surface. We approximate this by an image shift as explained in 37,39. The time-dependencies of the matrix elements $V_{\boldsymbol{k} \sigma}^{\mathrm{SET}}(t)$ for single electron transfer, $V_{\boldsymbol{k}_{1} \boldsymbol{k}_{2} \boldsymbol{k}^{\prime} \sigma \downarrow}^{\mathrm{AN}}(t)$ for Auger neutralization, $V_{\boldsymbol{k} \boldsymbol{k} / \sigma}^{\mathrm{DAD}}(t)$ for direct Auger de-excitation, and $V_{\boldsymbol{k} \boldsymbol{q} \sigma}^{\text {IAD }}(t)$ for indirect Auger deexcitation arise from the overlap of projectile and target wave functions which also depend on the separation of the projectile and the target. In our models the target is approximated by a three-dimensional potential step, its wave functions can thus be obtained analytically. For the bound states of the helium projectile we take hydrogen wave functions with charges adjusted to reproduce the ionization levels of helium. The projectile's continuum states are approximated by plane waves. Explicit expressions for the matrix elements will be given elsewhere 65 .

Subjecting model 10 to a quantum kinetic treatment along the lines initially given by Langreth and coworkers [60,61,62, a linear set of ordinary first order differential equations can be derived for the probabilities with which the projectile's electronic configurations occur in the course of the collision. In our case, we obtain equations for the occurrence probabilities of the ion, the groundstate, and the two metastable states of the helium projectile. The derivation consists of three main steps. First, twotime Dyson equations for the projectile Green functions are set up. Second, the selfenergies are calculated in the non-crossing approximation and, third, the fact is utilized that the selfenergies are peaked around the time-diagonal enabling a saddle-point approximation to extract from the Dyson equations rate equations for the occurrence probabilities $n_{i}(t), n_{t}(t), n_{s}(t)$, and $n_{g}(t)$ for, respectively, the ion, the two metastable states, and the groundstate of the projectile. Following this reasoning which we also exerted in 36, 38, 39, we get

$$
\frac{d}{d t}\left(\begin{array}{c}
n_{\mathrm{i}} \\
n_{\mathrm{t}} \\
n_{\mathrm{s}} \\
n_{\mathrm{g}}
\end{array}\right)=\left(\begin{array}{cccc}
-\left[\Gamma_{\mathrm{t}}^{<}(t)+\Gamma_{\mathrm{s}}^{<}(t)+\Gamma_{\mathrm{AN}}^{<}(t)\right] & \Gamma_{\mathrm{t}}^{>}(t) & \Gamma_{\mathrm{s}}^{>}(t) & 0 \\
\Gamma_{\mathrm{t}}^{<}(t) & -\left[\Gamma_{\mathrm{t}}^{>}(t)+\Gamma_{\mathrm{IAD} \uparrow}^{<}(t)\right] & 0 & 0 \\
\Gamma_{\mathrm{s}}^{<}(t) & 0 & -\left[\Gamma_{s}^{>}(t)+\Gamma_{\mathrm{IAD} \downarrow}^{<}(t)+\Gamma_{\mathrm{DAD} \downarrow}^{<}(t)\right] & 0 \\
\Gamma_{\mathrm{AN}}^{<}(t) & \Gamma_{\mathrm{IAD} \uparrow}^{<}(t) & \Gamma_{\mathrm{IAD} \downarrow}^{<}(t)+\Gamma_{\mathrm{DAD} \downarrow}^{<}(t) & 0
\end{array}\right) \cdot\left(\begin{array}{c}
n_{\mathrm{i}} \\
n_{\mathrm{t}} \\
n_{\mathrm{s}} \\
n_{\mathrm{g}}
\end{array}\right)
$$

which can be solved numerically. Explicit expressions for the time-dependent rates $\Gamma_{\ldots}^{\gtrless}(t)$ will be given elsewhere [65]. Notice, the entries in each column of the matrix sum up to zero. This is a consequence of the fact that the probability of finding the projectile at any particular instant of time in any one of its electronic configurations has to be one. 
Hence, $n_{i}(t)+n_{t}(t)+n_{s}(t)+n_{g}(t)=1$ implying

$$
\frac{d}{d t}\left[n_{i}(t)+n_{t}(t)+n_{s}(t)+n_{g}(t)\right]=0 .
$$

Representative data for the instantaneous occurrence probabilities $n_{i}(t), n_{s}(t), n_{t}(t)$ and $n_{g}(t)$ as well as the instantaneous secondary electron emission probability $\gamma_{e}(t)=$ $n_{g}(t) / 2$, assuming a fifty-fifty chance for the excited electron to leave the solid, obtained from the numerical solution of 111 are plotted in Figure 3 for a $\mathrm{He}^{+}\left(1^{2} \mathrm{~S}_{1 / 2}\right)$ ion hitting a tungsten surface with $E_{\text {kin }}=200 \mathrm{eV}$ under an angle of incident of $2^{\circ}$ measured from the surface. The parameter setting is not appropriate for a $\mathrm{He}^{+}\left(1^{2} \mathrm{~S}_{1 / 2}\right)$ ion scattering off a plasma wall where the incident would be normal and not grazing because of the sheath potential. However, the ion beam scattering experiments from which we obtained data for secondary electron emission coefficients have been performed for grazing incident 67. To compare our results with measured data we hence use this scattering geometry. Data for normal incident will be presented elsewhere 65. For the situation depicted in Fig. 3 the helium projectile thus starts as an ion at a distance $z=40 a_{\mathrm{B}}$ ( $a_{\mathrm{B}}$ is the Bohr radius), moves along a grazing trajectory towards the turning point $z_{\mathrm{tp}}=2.27 a_{\mathrm{B}}$, where it is specularly reflected to move back to the distance $z=40 a_{\mathrm{B}}$. After completion of the collision the projectile finds itself neutralized to the groundstate $\mathrm{He}\left(1^{1} \mathrm{~S}_{0}\right)$ with probability $n_{g}\left(t_{\max }\right) \approx 0.4$ and to the triplet metastable configuration $\mathrm{He}^{*}\left(2^{3} \mathrm{~S}_{1}\right)$ with probability $n_{t}\left(t_{\max }\right) \approx 0.3$. It thus survives the surface collision as an ion with probability $n_{i}\left(t_{\max }\right) \approx 0.3$.

The probability for emitting a secondary electron in the course of the collision is $\gamma_{e}\left(t_{\max }\right) \approx 0.2$ which agrees surprisingly well with experimental data by Müller and coworkers [67. Despite the simplicity of the model and the uncertainties arising from the Gadzuk construction [57,58. we use for obtaining the matrix elements of the Hamiltonian $(10)$, we nevertheless obtain very good results. Even the spectrum of the emitted electron (not shown) turns out to be in good agreement with the measured spectrum. The result for the helium ion is thus rather encouraging. It is of course not a hard test of the viability of the semiempirical approach. For that we should at least be also able to reproduce experimental data 67] for inert gas ions other than $\mathrm{He}^{+}\left(1^{2} \mathrm{~S}_{1 / 2}\right)$. Work in this direction is in progress. It requires the incorporation of $p$-orbitals and hence the renewed computation of all matrix elements and transition rates.

\section{Electric double layer}

Treating electron absorption/backscattering and electron extraction as elementary surface collision processes giving rise to a few surface parameters fed into the kinetic modeling of the plasma is a viable approach as long as the microphysics responsible for these processes is irrelevant on the scale of the plasma sheath. If this is however not the case, for instance, because of the miniaturization of the

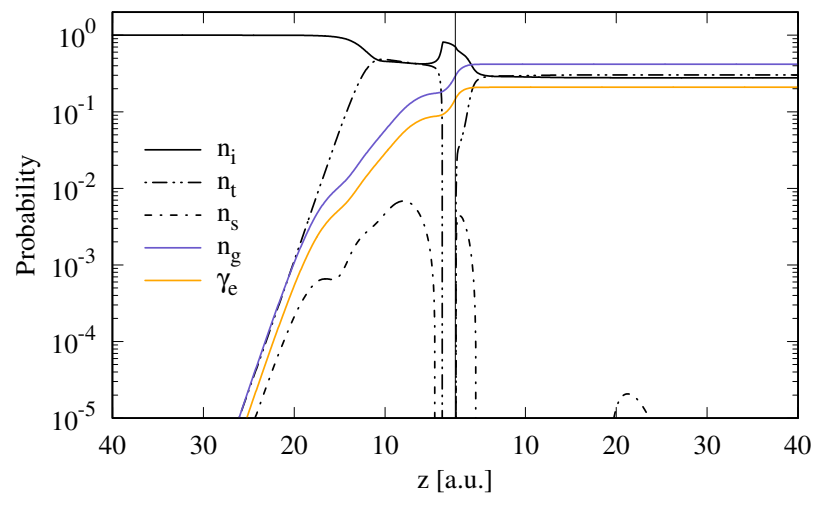

Fig. 3. (Color online) Instantaneous occurrence probabilities $n_{i}, n_{t}, n_{s}$, and $n_{g}$ of the electronic configurations of the helium projectile and instantaneous electron emission probability $\gamma_{e}$ obtained from (11). The projectile hits the tungsten surface as an ion with a kinetic energy $E_{\text {kin }}=200 \mathrm{eV}$ and an incident angle with respect to the surface of $\varphi=2^{\circ}$. The turning point $z_{\mathrm{tp}}=2.27$ and is indicated by the thin vertical line. Hence, the left (right) part of the plot denotes the incoming (outgoing) branch of the collision trajectory. At the end of the collision, the projectile is either still in its ionic state (black solid line), in its triplet metastable state (dot-dot-dashed line), or in its groundstate (blue solid line). The singlet metastable state (dotdashed line) occurs only temporarily. Its occurrence probability at the end of the collision is vanishingly small. The secondary electron emission coefficient is the value of $\gamma_{e}$ (orange solid line) at the end of the outgoing branch. It is in good agreement with experimental data [67.

discharges, or if the physical system of interest consists of a plasma and a solid component which cannot be separated and have thus to be treated on an equal footing, as it is the case for the plasma bipolar junction transistor [27, or if one is simply interested per se in how the electronic nonequilibrium of the plasma is transferred to the electronic states of the solid, one has to employ kinetic equations not only for the plasma but also for the solid and to merge them by suitable matching conditions. Improving our earlier work 41] on the distribution of surplus electrons accumulated from the plasma inside a plasma-facing dielectric we recently set up a general kinetic framework [40] for studying the charge transport across a dielectric plasma interface, that is, its electronic response, selfconsistently with the charge transport and relaxation on both sides of the interface.

Our first attempt 41] of treating the plasma-induced electric double layer at a dielectric plasma interface was based on a thermodynamic principle 68 and a graded potential 69 70 interpolating between the potential of the sheath and the potential inside the solid. Due to the graded potential we could treat dielectrics with positive and negative electron affinities. The assumption of thermodynamic equilibrium of the surplus charges with the lattice of the solid, however, is too restrictive. It applies only to a subgroup of transferred electrons. In the new approach 40] we overcome this limitation. The concept of an electron surface layer employed in 41 to describe 


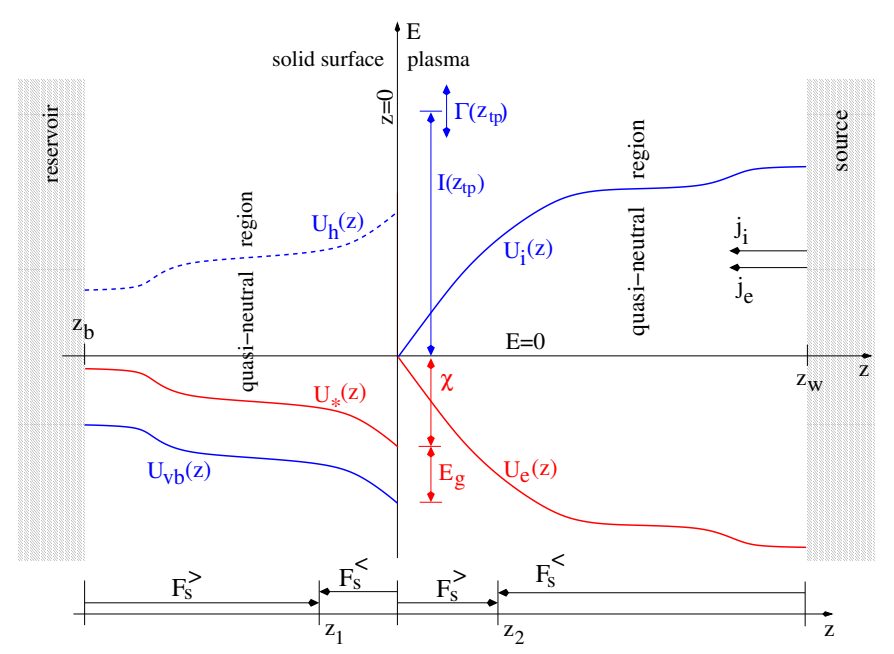

Fig. 4. (Color online) Interface model for an electric double layer with negative space charge inside the solid and positive space charge in front of it 40. Shown are the edges of the conduction $\left(U_{*}\right)$ and valence bands $\left(U_{\mathrm{vb}}\right)$, the edge for the motion of valence band holes $\left(U_{\mathrm{h}}\right)$, the potential energies for electrons and ions on the plasma side, and the energetic range, specified by the ion's ionization energy $I$ and its broadening $\Gamma$, in which hole injection occurs due to the neutralization of ions at the interface. Source, reservoir, and quasi-neutral regions are indicated as they will arise in the course of the calculation.

the solid-based negative part of the electric double layer enabled us however to determine in which states the electrons accumulated from the plasma reside: For dielectrics with negative electron affinity they are confined in image states in front of the surface, forming thus a twodimensional electron film as envisaged by Emeleus and Coulter [71,72 in their attempt to go beyond the perfect absorber model for plasma walls, while for electro-positive dielectrics they populate the surface's conduction band, giving thus rise to a wide space charge region.

The fundamental set of equations describing the electronic response of a plasma-facing dielectric solid is given in 40. It consists of the Poisson equation

$$
\frac{d}{d z} \varepsilon(z) \frac{d}{d z} U_{c}(z)=8 \pi \rho(z)=8 \pi\left[\rho_{w}(z) \theta(-z)-\rho_{p}(z) \theta(z)\right]
$$

and two sets of spatially separated Boltzmann equations, one for the electrons and ions in the plasma and one for the conduction band electrons and valence band holes in the solid. Defining a species index $s=e, i, *, h$ to denote electrons, ions, conduction band electrons, and valence band holes, respectively, the Boltzmann equations for the quasistationary distribution functions $F_{s}^{\gtrless}(z, E, \boldsymbol{K})$, for left and right moving particles (see Figure 4), can be written as

$$
\left[ \pm v_{s}(z, E, \boldsymbol{K}) \frac{\partial}{\partial z}+\gamma_{s}\left[F_{s^{\prime}}^{\gtrless}\right]\right] F_{s}^{\gtrless}(z, E, \boldsymbol{K})=\Phi_{s}\left[F_{s^{\prime}}^{\gtrless}\right]
$$

with

$$
v_{s}(z, E, \boldsymbol{K})=2\left(\frac{m_{e}}{m_{s}}\left[E-U_{s}(z)\right]-\left(\frac{m_{e}}{m_{s}} \boldsymbol{K}\right)^{2}\right)^{1 / 2}
$$

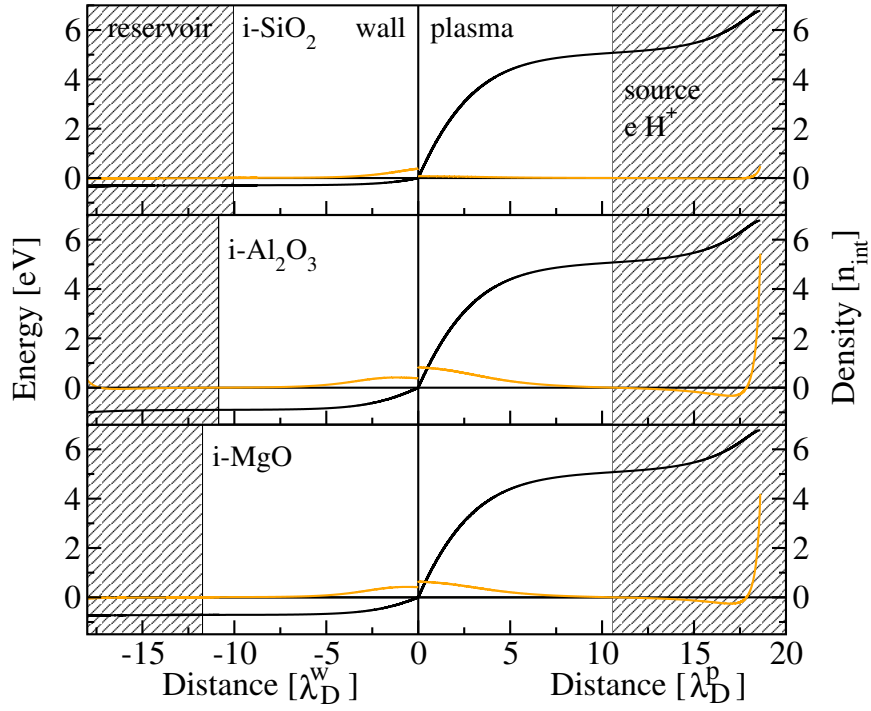

Fig. 5. (Color online) Potential energy (black lines) and density (orange lines) profiles defined by $\rho_{w}^{t}(z)=\left[n_{*}^{t}(z)-\right.$ $\left.n_{h}^{t}(z)\right] \theta(-z)$ and $\rho_{p}(z)=\left[n_{i}(z)-n_{e}(z)\right] \theta(z)$ for $\mathrm{i}_{-} \mathrm{SiO}_{2}$ [40, i$\mathrm{Al}_{2} \mathrm{O}_{3}$, and $\mathrm{i}-\mathrm{MgO}$ surfaces in contact with a hydrogen plasma with $k_{B} T_{e}=10 k_{B} T_{i}=2 \mathrm{eV}$. Only the thermalized part of the charge density inside the wall, $\rho_{w}^{t}(z)$, is shown which balances $\rho_{p}(z)$ on the plasma side. The interface is assumed to be perfectly absorbing and collisionless on both sides. Grey regions on the left and right indicate respectively the reservoir and the source required for implementing the physical boundary conditions for the electric double layer. Relevant for the double layer is only the region between the two thin vertical lines indicating the positions of the inflection points $z_{1}<0$ and $z_{p}>0$ in units of the Debye screening lengths $\lambda_{D}^{w}$ and $\lambda_{D}^{p}$, respectively. The band bending is given by $U_{1}=U_{c}\left(z_{1}\right)$ while the sheath potential becomes $U_{p}=U_{c}\left(z_{p}\right)$. Material parameters used for the calculations are summarized in Table 1

the velocity of the particles normal to the (planar) interface at $z=0$ and $z, E$, and $\boldsymbol{K}$ the distance from the interface, the total energy, and the lateral momentum. The potential energies entering (15) are given by

$$
\begin{aligned}
& U_{*}(z)=-U_{c}(z)-\chi, \quad U_{e}(z)=-U_{c}(z), \\
& U_{h}(z)=U_{c}(z)+E_{g}+\chi, \quad U_{i}(z)=U_{c}(z)
\end{aligned}
$$

with $U_{c}(z)$ the solution of $(13), E_{g}$ the band gap, and $\chi>0$ the electron affinity. Energies and lengths are again measured in Rydbergs and Bohr radii.

The functions $\gamma_{s}\left[F_{s^{\prime}}^{\gtrless}\right]$ and $\Phi_{s}\left[F_{s^{\prime}}^{\gtrless}\right]$ denote, respectively, the rates for out-scattering and the in-scattering collision integrals. The set of Boltzmann equations has to be augmented by matching conditions for the distribution functions. For electrons they can be derived quantummechanically using a technique developed for solid surfaces 73 and solid-solid heterostructures 74,75 . So far the technique has been applied to match bulk electron states across interfaces. In principle it should be however possible to generalize the approach to take surface states into account as well. The matching of the ion and hole distribution functions on the other hand has to be based on a physical model for hole injection due to, for instance, 


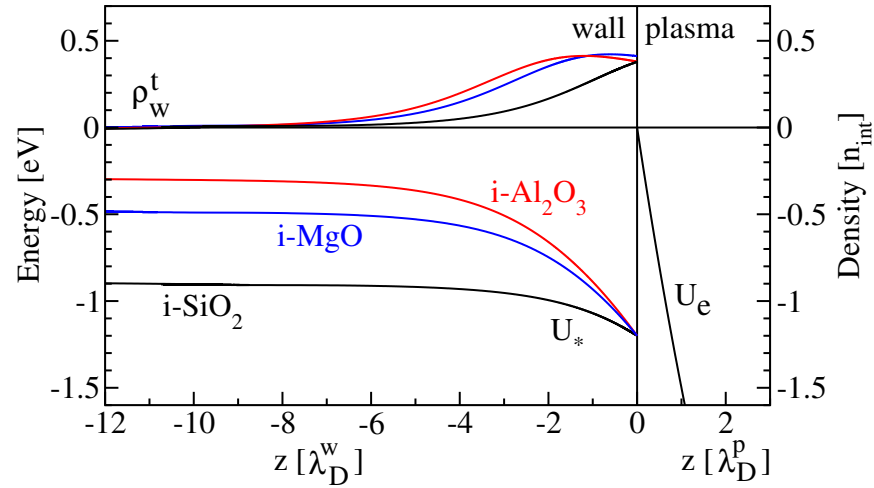

Fig. 6. (Color online) Conduction band edges $U_{*}(z)$ and density profiles $\rho_{w}^{t}(z)$ for i-SiO $2, \mathrm{i}-\mathrm{Al}_{2} \mathrm{O}_{3}$, and $\mathrm{i}-\mathrm{MgO}$ surfaces in contact with an hydrogen plasma with $k_{B} T_{e}=10 k_{B} T_{i}=2 \mathrm{eV}$. Material parameters can be found in Table 1 . The interface is perfectly absorbing and collisionless on both sides.

resonant neutralization of ions at the interface. Neutralization due to Auger processes leading in addition to the emission of a secondary electron as discussed in Section 3 could be also treated but we have not done so yet.

For a dielectric plasma interface without surface states and for resonant ion neutralization only the matching conditions for the distribution functions become 40 .

$$
\begin{aligned}
F_{e, *}^{>,<}(0, E, \boldsymbol{K}) & =R(E, \boldsymbol{K}) F_{e, *}^{<,>}(0, E, \boldsymbol{K}) \\
& +[1-R(E, \boldsymbol{K})] F_{*, e}^{>,<}(0, E, \boldsymbol{K})
\end{aligned}
$$

with $E>0$ and

$$
\begin{aligned}
& F_{h}^{<}(0, E, \boldsymbol{K})=F_{h}^{>}(0, E, \boldsymbol{K})+\alpha S_{h}^{<}(E, \boldsymbol{K}), \\
& F_{i}^{>}(0, E, \boldsymbol{K})=(1-\alpha) F_{i}^{<}(0, E, \boldsymbol{K})
\end{aligned}
$$

with $E>E_{g}+\chi$, where $R(E, \boldsymbol{K})$ is the quantum mechanical reflection coefficient for electrons due to the surface potential, $\alpha$ is the ion neutralization probability, and $S_{h}^{<}(E, \boldsymbol{K})$ is a function specifying hole injection into the valence band of the dielectric due to the neutralization of an ion at the interface. The particular form of $S_{h}^{<}(E, \boldsymbol{K})$ depends on the neutralization process. In case the neutralization induces also secondary electron emission, the matching condition for the electron distribution functions has to be augmented by a function $S_{e}^{>}(E, \boldsymbol{K})$. The functions $S_{h}^{<}(E, \boldsymbol{K})$ and $S_{e}^{>}(E, \boldsymbol{K})$ have thus to account for the processes discussed in Section 3 .

Together with the Poisson equation 13 for the electric potential energy $U_{c}(z)$, the matching conditions for $U_{c}(z)$ at $z=0$, and

$$
n_{s}(z)=\int \frac{d E d^{2} K}{(2 \pi)^{3}} \frac{F_{s}^{>}(z, E, \boldsymbol{K})+F_{s}^{<}(z, E, \boldsymbol{K})}{v_{s}(z, E, \boldsymbol{K})},
$$

for the charge densities, Eqs. 14 - 20 form a closed set of equations for the distribution functions provided they are supplemented by boundary conditions far away from the interface, that is, at $z=z_{b}<0$ and $z=z_{w}>0$ (see Figure 4). For a floating plasma interface the boundary condition at $z=z_{w}$ is the quasi-neutral bulk plasma, acting as a source for ions and electrons, whereas at $z=z_{b}$ an intrinsic or extrinsic dielectric has to be established as a quasi-neutral reservoir for conduction band electrons and valence band holes. The physical picture arising for a floating interface is illustrated in Figure 4 anticipating an electron-rich (electron-depleted) surface (plasma sheath). Electrically contacted plasma interfaces (electrodes) require other boundary conditions and have to be modelled differently.

The approach presented so far is quite general. To obtain results scattering processes have to be specified. On the plasma side the most important scattering process is charge exchange scattering for ions whereas inside the solid intraband electron-phonon scattering and interband electron-hole recombination should be included. Once the collision integrals associated with these processes are worked out the set of Boltzmann equations has to be solved numerically together with the Poisson equation, the matching conditions, and the boundary conditions. This is quite demanding and part of our future activities in this field.

To obtain first estimates for the electric double layer forming due to the electronic response of the interface, we considered in 40 the case of a collisionless, perfectly absorbing interface. In this particular case the lateral momentum $\boldsymbol{K}$ can be eliminated. It is then possible to formulate the whole approach in terms of

$$
F_{s}(z, k)=\int \frac{d^{2} K}{(2 \pi)^{2}} F_{s}(z, \boldsymbol{K}, k),
$$

which satisfy

$$
\pm v_{s}(z, E) \frac{\partial}{\partial z} F_{s}^{\gtrless}(z, E)=0
$$

with

$$
v_{s}(z, E)=2\left(\frac{m_{e}}{m_{s}}\left[E-U_{s}(z)\right]\right)^{1 / 2} .
$$

The Boltzmann equations become thus ordinary differential equations which can be solved, as explained in 40, by a straightforward trajectory analysis taking at $z=0$ the perfect absorber matching conditions and at $z=z_{b, w}$ the boundary conditions into account specified in the previous paragraph. For details see 40. It turns out that in this particular case the densities $n_{s}(z)$ are only functions of $U_{c}(z)$ and not of $z$ explicitly. Hence, the Poisson equation can be integrated once analytically and a second time numerically to obtain $U_{c}(z)$.

Numerical results for this simple case are shown in Figures 5 and 6 using a hydrogen plasma in contact with intrinsic $\mathrm{SiO}_{2}, \mathrm{Al}_{2} \mathrm{O}_{3}$, and $\mathrm{MgO}$ surfaces as examples. The material parameters are given in Table 1. Notice in particular the plasma-induced band bending inside the dielectrics. As in other electronic devices, it would control together with the band off-set $\chi$ the electric current in case the interface was wired up to an external circuit. To make the approach selfconsistent, it is necessary to 


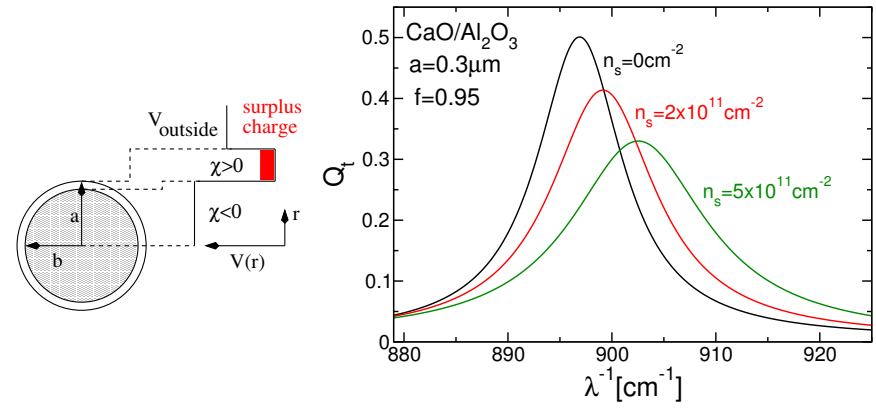

Fig. 7. (Color online) Geometry and profile of the conduction band edges for a $\mathrm{CaO} / \mathrm{Al}_{2} \mathrm{O}_{3}$ core-shell particle confining surplus electrons accumulated from the plasma in a narrow shell of thickness $d=(1-f) a$ with $f=b / a$ the filling factor. The antibonding resonance arising from the surface modes at the two interfaces shifts with charge density (data adapted from 42.)

split the charge density inside the solid into a thermalized (trapped) and a non-thermalized (non-trapped) part: $\rho_{w}(z)=\rho_{w}^{t}(z)+\rho_{w}^{j}(z)$. Roughly speaking, $\rho_{w}^{t}(z)$ is a consequence of the boundary condition at $z=z_{b}$ while $\rho_{w}^{j}(z)$ is a consequence of the matching condition at the interface. In effect this leads to a two-species scenario and a recombination condition limiting in conjunction with the boundary conditions the influx $j=j_{e}=j_{i}$ of electrons and ions to the interface. Numerical data for the influx are given with other quantities of interest in Table 2, Notice, even in the simply case considered here-the collisionless, perfectly absorbing plasma interface-the influx $j$ and the total wall charge $N_{\mathrm{w}}=\int_{z_{1}}^{0} d z \rho_{w}^{t}(z)$ establishing quasi-stationarity depend on the material. The reason is the selfconsistent adjustment of the plasma source to the environment inside the solid characterized here by the intrinsic charge density $n_{\text {int }}$ (given in Table 2), the energy gap $E_{g}$, and the temperatures of the thermalized holes and electrons, $T_{h}$ and $T_{*}$. In a realistic model, where collisions are included, in particular, interband collisions annihilating electron-hole pairs, the adjustment of the plasma source to the conditions inside the wall will become even more pertinent.

\section{Spectroscopy of the wall charge}

In an effort to establish a method for measuring the charge of dust grains in a plasma optically we investigated Mie scattering by charged dielectric particles. Initially we considered homogeneous particles with negative and positive electron affinity [43,44 but later we also investigated coreshell particles, where an electro-negative core is coated by an electro-positive film 42. The idea was based on the observation that the particle charge modifies either the boundary condition for the electromagnetic field (particles with negative electron affinity) or the bulk dielectric function (particles with positive electron affinity). In either case the optical extinction $Q_{t}$ depends on the charge of the particle which should thus be visible in the Mie signal calculated and measured by standard procedures [76].
We found light scattering by particles made out of dielectric materials featuring transverse optical phonons, for instance, $\mathrm{MgO}, \mathrm{CaO}, \mathrm{Al}_{2} \mathrm{O}_{3}$ to be very charge sensitive in the vicinity of anomalous optical resonances 43 , 44, defined by $\varepsilon^{\prime}(\omega)<0$ and $\varepsilon^{\prime \prime}(\omega) \ll 1$, where $\varepsilon(\omega)=$ $\varepsilon^{\prime}(\omega)+i \varepsilon^{\prime \prime}(\omega)$ is the complex dielectric function of the material 77,78 . The resonances are in the infrared and shift with increasing charge towards higher wave numbers. Confining the charge to a narrow electro-positive shell around an electro-negative core increases the charge sensitivity of the shift [42. In Figure 7 the geometry of such a composite particle is shown together with results for a $\mathrm{CaO} / \mathrm{Al}_{2} \mathrm{O}_{3}$ core-shell particle. It turns out 79 that the antibonding resonance, arising from the hybridization of the two surface modes 80,81 localized, respectively, at the core-shell and the shell-vacuum interface (that is, in plasma applications, the shell-plasma interface) is most charge-sensitive. Core-shell particles with this morphology could thus be used as electric probes with optical read-out.

The idea of using engineered solid-state objects as electric probes can be also applied to planar walls. Indeed, the surface modes responsible for the charge-sensitivity of Mie scattering by small particles become the surface-plasmon polaritons in the case of planar walls. Their dispersion and damping depends on the dielectric function of the wall which in turn is modified by the surplus electrons accumulated from the plasma. Spectroscopic techniques sensitive to the surface-plasmon polariton should thus be capable to reveal information about the space charge inside the solid. Hence, using such a spectroscopy in plasma applications would provide a sensor for the physical properties of the wall charge. Particularly promising in this respect seems to be electron-energy loss spectroscopy 82,83 , which demonstrated for free-standing surfaces and solid heterostructures its feasibility for investigating buried space charge regions $84,85,86$.

An exploratory theoretical study of small-angle inelastic electron scattering on a dielectric heterostructure consisting of a charge-carrying layer of thickness $d_{1}$, in contact with a plasma, and a substrate layer of thickness $d_{2}$ showed that the charge dependence of the effective dielectric function governing the scattering of the electron is strong enough to produce measurable effects also in cases where the electron beam is applied to the substrate layer and not to the charge-carrying layer as shown in the left panel of Figure 8. Similar to Mie scattering by charged dielectric core-shell particles 42 the antibonding resonance arising from the two surface modes at, respectively, the dielectric/dielectric and the dielectric/plasma interface turns out to be particularly charge sensitive. The charge-dependent shift of its spectral position is shown on the right side of Figure 8 . It is the larger the thinner the charge-carrying layer, that is, the stronger the surplus electrons are confined.

To obtain the data shown in Figure 8 we assumed electrons with surface density $n_{s}$ to be homogeneously distributed in the charge-carrying layer and calculated, using $n_{s}, d_{1}$, and $d_{2}$ as parameters, the electron energy loss 

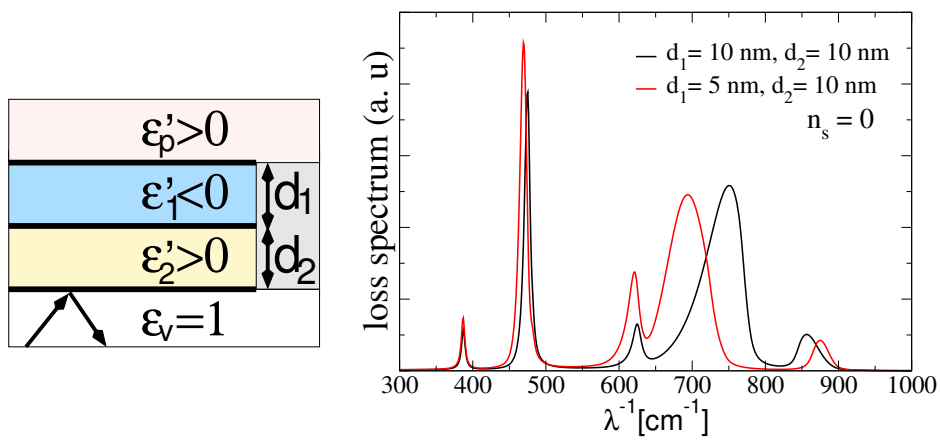

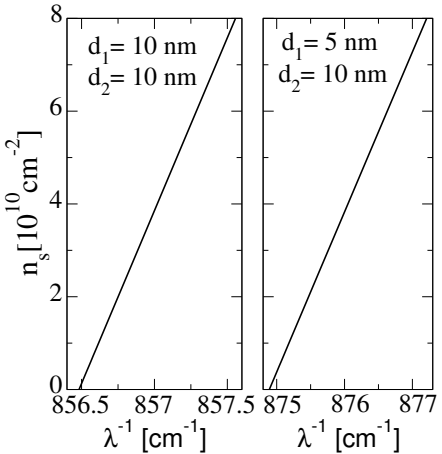

Fig. 8. (Color online) Results for the electron energy loss spectrum of a stack of two dielectric layers in contact with a plasma providing the dielectric mismatch at the uppermost interface and the surplus charges inside the plasma-facing layer (although the charge transfer from the plasma to the layer is not explicitly modelled). The charge-carrying layer has thickness $d_{1}$ while the substrate has thickness $d_{2}$. The most charge-sensitive feature of the loss spectrum is the peak in the range $850-870 \mathrm{~cm}^{-1}$ which is the antibonding resonance arising from the modes at the dielectric/dielectric and the dielectric/plasma interface. Its shift with charge density is plotted on the right for two different thicknesses $d_{1}$ of the charge-carrying layer.

spectrum 83 ,

$$
\frac{d S}{d \omega}=\int d^{2} q_{\|} A\left(\boldsymbol{q}_{\|}, \omega\right) \operatorname{Im}\left[\frac{-1}{\varepsilon_{\mathrm{eff}}\left(\boldsymbol{q}_{\|}, \omega\right)+1}\right],
$$

where the scattering kinetics is accounted for by the function

$$
A\left(\boldsymbol{q}_{\|}, \omega\right)=\frac{4 e^{2}}{\pi^{2} \hbar} \frac{q_{\|} v_{\perp}^{2}}{\left[\left(\omega-\boldsymbol{q}_{\|} \cdot \boldsymbol{v}_{\|}\right)^{2}+q_{\|}^{2} v_{\perp}^{2}\right]^{2}},
$$

with $\boldsymbol{v}_{\|}$and $v_{\perp}$ the lateral and perpendicular velocity of the electron with respect to the interface, and the energy loss is encoded into an effective dielectric function

$$
\varepsilon_{\mathrm{eff}}\left(\boldsymbol{q}_{\|}, \omega\right)=a_{2}-\frac{b_{2}^{2}}{a_{2}+a_{1}-b_{1}^{2} /\left(a_{1}+a_{p}\right)}
$$

with $a_{i}=\varepsilon_{i} \operatorname{coth}\left(q_{\|} d_{i}\right)$ and $b_{i}=\varepsilon_{i} / \sinh \left(q_{\|} d_{i}\right)$ for $i=$ $1,2, p$ and $b_{p}=0$. Neglecting nonlocal effects, the surplus charges affect the effective dielectric function only through the dielectric function of the charge-carrying layer,

$$
\varepsilon_{1}(\omega) \rightarrow \tilde{\varepsilon}_{1}(\omega)=\varepsilon_{1}(\omega)+4 \pi i \sigma(\omega) / \omega,
$$

where $\sigma(\omega)$ is the phonon-limited conductivity of a gas of electrons with volume density $n_{b}=n_{s} / d_{1}$ to be obtained in the same way as in 42 .

The numerical results plotted in Figure 8, obtained for a charge-carrying layer made out of $\mathrm{Al}_{2} \mathrm{O}_{3}$ and a dissipationless substrate layer specified by $\varepsilon_{2}=2$, demonstrate that the charge-induced modification of the effective dielectric function can be strong enough to be detectable by a scattering geometry where the beam of electrons suffering energy loss is not in direct contact with the charge-carrying layer. This is important if one wants to employ electron energy loss spectroscopy as a probe for the charge build-up in the wall of a bounded plasma where the charged species and the electric fields in the plasma prevent the probing electron beam to be applied to the plasma-facing side of the wall. To measure the charge distribution $\rho_{w}(z)$ accumulated in a plasma-facing solid structure can thus not be performed in the (standard) scattering geometry developed, for instance, for investigating space charge layers at semiconducting surfaces 84 , 85, 86. Our results demonstrate however that from-theback diagnostics of $\rho_{w}(z)$ or $N_{\mathrm{w}}$, suitable for plasma applications, should be feasible.

\section{Concluding remarks}

In a still on-going effort we work towards a microscopic understanding of the electron kinetics across the plasma interface. The main motivation for our work, which we reviewed and extended in this presentation in a colloquial manner-the mathematical details of which can be found in the original publications or in the work to be publishedstems from the prospects of solid-based integrated microdischarges. We expect the charge transfer between a solid and a plasma to be controllable by a judicious design of the plasma-facing structure opening up thereby routes to a new generation of opto-electronic plasma devices. Even if this expectation does not become reality conventional discharges also benefit from a better understanding of the charge transfer between the plasma and the solid because it leads to more realistic electron absorption/backscattering and secondary electron emission coefficients.

As far as electron absorption/backscattering and electron extraction due to atomic particles as elementary surface collision processes are concerned we presented general theoretical frameworks for calculating electron sticking/backscattering and secondary electron emission probabilities. In particular the invariant embedding approach used for investigating the interaction of a low-energy electron with an electro-positive dielectric surface has great potential. It can be extended to higher electron energies, above the impact ionization threshold of the dielectric, and also applied to metallic surfaces, if it is augmented by Coulomb-driven backscattering processes. The semiem- 
pirical multi-channel Anderson-Newns model we use for calculating secondary electron emission probabilities due to low-energy atom-surface collisions (potential ejection of electrons) leads to very promising results for the projectiletarget combinations we applied it to despite the uncertainties of the Gadzuk construction used for the calculation of the matrix elements. Further studies along this line, and comparison with results obtained from more refined treatments, are however necessary to demonstrate that the semiempirical model produces indeed viable results for potential electron ejection due to charge-transferring atom-surface collisions. Kinetic ejection of electrons, occurring at impact energies on the order of $100 \mathrm{eV}$, has to be treated differently, because the projectile has then enough energy to enter the solid leading then to electron emission due to a cascade of collisions inside the solid.

We also presented the fundamental set of equations describing at the semiclassical level the selfconsistent electronic response of a plasma-facing solid. It consists of the Poisson equation for the electric potential energy and two sets of spatially separated Boltzmann equations, one for the electrons and ions inside the plasma and one for the conduction band electrons and valence band holes inside the solid. The solutions of the two sets of Boltzmann equations are connected by matching conditions. For electrons the matching conditions describe quantum-mechanical reflection and transmission due to the abrupt change in potential energy at the plasma interface whereas for ions and holes the matching conditions mimic hole injection (and thus electron extraction) due to resonant neutralization of ions. Secondary electron emission due to high energy electrons impacting the surface and/or Auger neutralization (de-excitation) of ions (metastable states) could be also included into the matching conditions. In an exploratory investigation we applied the approach to a perfectly absorbing collisionless plasma interface and obtained first estimates for the plasma-induced band bending and the spatial charge distribution of the solid-based part of the electric double layer. Making the interface model more realistic by going beyond the perfect absorber assumption, accounting for collisions on both sides of and at the interface, and including the full band structure of the surface is of course necessary to produce reliable data. All this can be however done within the general framework presented above resulting in a kinetic model revealing-for the first time-the basic mechanisms coupling the charge production on the gaseous side of the interface with the charge losses inside the solid.

Based on our proposal of using spectroscopic means to determine the charge of a micron-size dust particle in a plasma we explored furthermore the possibility to employ electron-energy loss spectroscopy to probe the charge accumulated in a planar plasma-facing solid. Having experimental access to the solid-based negative part of the electric double layer at a plasma interface will be crucial for developing a quantitative microscopic understanding of the charge transfer across the interface. Our preliminary results indicate electron-energy loss spectroscopy to be indeed sensitive to the charge distribution inside plasma- facing solid structures. We expect it thus to be a key experimental technique for quantifying plasma-induced space charge layers in solids.

Support from the Deutsche Forschungsgemeinschaft through Project B10 of the second and third funding period of the Transregional Collaborative Research Center SFB/TRR 24 is greatly acknowledged. The contributions of R. L. Heinisch and J. Marbach in the second funding period are also greatly acknowledged.

\section{Author contribution statement}

F. X. B. and H. F. outlined the scope and strategy of the calculations on which the work presented here is based; the computations of the electron sticking and backscattering coefficients as well as the calculations devoted to the electric double layer were performed by F. X. B.; M. P. and E. T. performed, respectively, the calculations concerning the neutralization of helium ions and the spectroscopy of the wall charge. The paper was written by F. X. B.; all authors contributed to the work presented here and approved the final text of the manuscript.

\section{References}

1. I. Langmuir, H. Mott-Smith, Gen. Electr. Rev. 27, 449 (1924)

2. J.G. Andrews, J.E. Allen, Proc. Roy. Soc. Lond. A 320, 459 (1971)

3. H. Schamel, S. Bujarbarua, Phys. Fluids 26, 190 (1983)

4. C. Charles, Plasma Sources Sci. Technol. 16, R1 (2007)

5. M.A. Raadu, Phys. Reports 178, 25 (1989)

6. R.T. Tung, Material Science and Engineering R35, 1 (2001)

7. S. Robertson, Plasma Phys. Control. Fusion 55, 093001 (2013)

8. R.N. Franklin, J. Phys. D: Appl. Phys. 36, R309 (2003)

9. K.U. Riemann, J. Phys. D: Appl. Phys. 24, 493 (1991)

10. M.D. Campanell, M.V. Umansky, Phys. Rev. Lett. 116, $085003(2016)$

11. D. Sydorenko, I. Kaganovich, Y. Raitses, A. Smolyakov, Phys. Rev. Lett. 103, 145004 (2009)

12. P.N. Hu, S. Ziering, Phys. Fluids 9, 2168 (1966)

13. R.N. Franklin, Plasma phenomena in gas discharges (Clarendon Press, Oxford, 1976)

14. D. Uhrlandt, M. Schmidt, J.F. Behnke, T. Bindemann, J. Phys. D: Appl. Phys. 33, 2475 (2000)

15. Y.B. Golubovskii, V.A. Maiorov, J. Behnke, J.F. Behnke, J. Phys. D: Appl. Phys. 35, 751 (2002)

16. H. Kersten, H. Deutsch, G.M.W. Kroesen, Int. J. Mass Spectr. 233, 51 (2004)

17. M.J. Kushner, J. Phys. D: Appl. Phys. 38, 1633 (2005)

18. D. Loffhagen, F. Sigeneger, Plasma Sources Sci. Technol. 18, 034006 (2009)

19. M. Shihab, A.T. Elgendy, I. Korolov, A. Derzsi, J. Schulze, D. Eremin, T. Mussenbrock, Z. Donkó, R.P. Brinkmann, Plasma Sources Sci. Technol. 22, 055013 (2013)

20. P. Tolias, Plasma Phys. Control. Fusion 56, 123002 (2014) 
21. V.I. Demidov, S.F. Adams, I.D. Kaganovich, M.E. Koepke, I.P. Kurlyandskaya, Phys. Plasma 22, 104501 (2015)

22. M. Daksha, B. Berger, E. Schuengel, I. Korolov, A. Derzsi, M. Koepke, Z. Donkó, J. Schulze, J. Phys. D: Appl. Phys. 49, $234001(2016)$

23. A. Marcak, C. Corbella, T. de los Arcos, A. von Keudell, Rev. Sci. Instrum. 86, 106102 (2015)

24. M.K. Kulsreshath, L. Schwaederle, L.J. Overzet, P. Lefaucheux, J. Ladroue, T. Tillocher, O. Aubry, M. Woytasik, G. Schelcher, R. Dussart, J. Phys. D: Appl. Phys. 45, 285202 (2012)

25. R. Dussart, L.J. Overzet, P. Lefaucheux, T. Dufour, M. Kulsreshath, M.A. Mandra, T. Tillocher, O. Aubry, S. Dozias, P. Ranson et al., Eur. Phys. J. D 60, 601 (2010)

26. J.G. Eden, S.J. Park, J.H. Cho, M.H. Kim, T.J. Houlahan, B. Li, E.S. Kim, T.L. Kim, S.K. Lee, K.S. Kim et al., IEEE Trans. Plasma Sci. 41, 661 (2013)

27. C.J. Wagner, P.A. Tchertchian, J.G. Eden, Appl. Phys. Lett. 97, 134102 (2010)

28. F.J.J. Peeters, M.C.M. van de Sanden, Plasma Sources Sci. Technol. 24, 015016 (2015)

29. R. Tschiersch, M. Bogaczyk, H.E. Wagner, J. Phys. D: Appl. Phys. 47, 365204 (2014)

30. M. Bogaczyk, R. Wild, L. Stollenwerk, H.E. Wagner, J. Phys. D: Appl. Phys. 45, 465202 (2012)

31. F.X. Bronold, H. Fehske, Plasma Phys. Control. Fusion 59, 014011 (2017)

32. F.X. Bronold, H. Fehske, Phys. Rev. Lett. 115, 225001 (2015)

33. R.L. Heinisch, F.X. Bronold, H. Fehske, Phys. Rev. B 83, 195407 (2011)

34. R.L. Heinisch, F.X. Bronold, H. Fehske, Phys. Rev. B 82, 125408 (2010)

35. R.L. Heinisch, F.X. Bronold, H. Fehske, Phys. Rev. B 81, $155420(2010)$

36. J. Marbach, F.X. Bronold, H. Fehske, Phys. Rev. B 86, $115417(2012)$

37. J. Marbach, F.X. Bronold, H. Fehske, Eur. Phys. J. D 66 , $106(2012)$

38. M. Pamperin, F.X. Bronold, H. Fehske, Phys. Scr. T165, 014008 (2015)

39. M. Pamperin, F.X. Bronold, H. Fehske, Phys. Rev. B 91, $035440(2015)$

40. F.X. Bronold, H. Fehske, J. Phys. D: Appl. Phys. 50 294003 (2017)

41. R.L. Heinisch, F.X. Bronold, H. Fehske, Phys. Rev. B 85, 075323 (2012)

42. E. Thiessen, R.L. Heinisch, F.X. Bronold, H. Fehske, Eur. Phys. J. D 68, 98 (2014)

43. R.L. Heinisch, F.X. Bronold, H. Fehske, Phys. Rev. E 88, 023109 (2013)

44. R.L. Heinisch, F.X. Bronold, H. Fehske, Phys. Rev. Lett. 109, $243903(2012)$

45. H.J. Kreuzer, Z.W. Gortel, Physisorption Kinetics (Springer Verlag, Berlin, 1986)

46. Z.W. Gortel, H.J. Kreuzer, R. Teshima, Phys. Rev. B 22, 5655 (1980)

47. J. Cazaux, J. Appl. Phys. 111, 064903 (2012)

48. R. Dashen, Phys. Rev. 134, A1025 (1964)

49. M. Vicanek, Surf. Sci. 440, 1 (1999)

50. L.G. Glazov, I. Pázsit, Nucl. Instr. and Meth. B 256, 638 (2007)
51. D.L. Smith, E.Y. Lee, V. Narayanamurti, Phys. Rev. Lett. 80, 2433 (1998)

52. C.J. Cook, W.J. Fredericks, J. Chem. Phys 36, 608 (1962)

53. G.F. Dionne, J. Appl. Phys. 46, 3347 (1975)

54. A. V. Phelps and Z. Lj Petrović, Plasma Sources Sci. Technol. 8, R21 (1999)

55. J. Marbach, F.X. Bronold, H. Fehske, Phys. Rev. B 84, $085443(2011)$

56. A. Yoshimori, K. Makoshi, Prog. Surf. Sci. 21, 251 (1986)

57. J.W. Gadzuk, Surface Science 6, 133 (1967)

58. J.W. Gadzuk, Surface Science 6, 159 (1967)

59. P. Coleman, Phys. Rev. B 29, 3035 (1984)

60. D.C. Langreth, P. Nordlander, Phys. Rev. B 43, 2541 (1991)

61. H. Shao, D.C. Langreth, P. Nordlander, Phys. Rev. B 49, 13929 (1994)

62. H. Shao, D.C. Langreth, P. Nordlander, in Low energy ionsurface interaction, edited by J.W. Rabalais (Wiley and Sons, New York, 1994), p. 117

63. X. He, J.A. Yarmoff, Phys. Rev. Lett. 105, 176806 (2010)

64. X. He, J.A. Yarmoff, Nucl. Instrum. Meth. Phys. Res. B 269, 1195 (2011)

65. M. Pamperin, F.X. Bronold, H. Fehske, unpublished (2017)

66. C.E. Sosolik, J.R. Hampton, A.C. Lavery, B.H. Cooper, J.B. Marston, Phys. Rev. Lett. 90, 013201 (2003)

67. H. Müller, R. Hausmann, H. Brenten, A. Niehaus, V. Kempter, Z. Phys. D 28, 109 (1993)

68. E.E. Tkharev, A.L. Danilyuk, Vacuum 35, 183 (1985)

69. F. Stern, Phys. Rev. B 17, 5009 (1978)

70. F. Stern, S.D. Sarma, Phys. Rev. B 30, 840 (1984)

71. K.G. Emeleus, J.R.M. Coulter, Int. J. Electronics 62, 225 (1987)

72. K.G. Emeleus, J.R.M. Coulter, IEE Proceedings 135, 76 (1988)

73. L.A. Falkovsky, Adv. Phys. 32, 753 (1983)

74. V.K. Dugaev, V.I. Litvinov, P.P. Petrov, Phys. Rev. B 52, 5306 (1995)

75. D. Schroeder, J. Appl. Phys. 72, 964 (1992)

76. C.F. Bohren, D.R. Huffman, Absorption and scattering of light by small particles (Wiley, 1983)

77. M.I. Tribelsky, B.S. Luk'yanchuk, Phys. Rev. Lett. 97, 263902 (2006)

78. M.I. Tribelsky, Europhys. Lett. 94, 14004 (2011)

79. E. Thiessen, R.L. Heinisch, F.X. Bronold, H. Fehske, Phys. Rev. A 93, 033827 (2016)

80. E. Prodan, C. Radloff, N.J. Halas, P. Nordlander, Science 302, 419 (2003)

81. T.C. Preston, R. Signorell, Proc. Nat. Acad. U. S. Sci 302, 5532 (2003)

82. H. Ibach and D. L. Mills, Electron energy loss spectroscopy and surface vibrations (Academic Press, New York, 1982)

83. Ph. Lambin and J. P. Vigneron and A. A. Lucas, Phys. Rev. B 32, 8203 (1985)

84. I. Mahboob, T.D. Veal, C.F. McConville, H. Lu, W.J. Schaff, Phys. Rev. Lett. 92, 036804 (2004)

85. H. Lüth, Vacuum 38, 223 (1988)

86. D. H. Ehlers and D. L. Mills, Phys. Rev. B 36, 1051 (1987) 


\begin{tabular}{|l||lll||lll||lll|}
\hline & $\begin{array}{l}\lambda_{D}^{w} \\
\left(10^{-3} \mathrm{~cm}\right)\end{array}$ & $\begin{array}{l}U_{1} \\
(\mathrm{eV})\end{array}$ & $\begin{array}{l}U_{b} \\
(\mathrm{eV})\end{array}$ & $\begin{array}{l}\lambda_{D}^{p} \\
\left(10^{-3} \mathrm{~cm}\right)\end{array}$ & $\begin{array}{l}U_{p} \\
(\mathrm{eV})\end{array}$ & $\begin{array}{l}U_{w} \\
(\mathrm{eV})\end{array}$ & $\begin{array}{l}n_{\text {int }} \\
\left(10^{10} \mathrm{~cm}^{-3}\right)\end{array}$ & $\begin{array}{l}N_{\mathrm{w}} \\
\left(10^{7} \mathrm{~cm}^{-2}\right)\end{array}$ & $\begin{array}{l}j \\
\left(10^{15} \mathrm{~s}^{-1} \mathrm{~cm}^{-2}\right)\end{array}$ \\
\hline $\mathrm{i}-\mathrm{SiO}_{2}$ & 2.4 & -0.3 & -0.4 & 6.3 & 5.1 & 6.8 & 7.5 & 15.2 & 3.5 \\
$\mathrm{i}-\mathrm{Al}_{2} \mathrm{O}_{3}$ & 3.2 & -0.9 & -1 & 1.6 & 5.1 & 6.8 & 10.7 & 59.6 & 56.7 \\
$\mathrm{i}-\mathrm{MgO}$ & 1.1 & -0.7 & -0.8 & 0.6 & 5.1 & 6.8 & 92.4 & 156.0 & 377.6 \\
\hline
\end{tabular}

Table 2. Numerical data for the electric double layer formed, respectively, at an intrinsic $\mathrm{SiO}_{2}, \mathrm{Al}_{2} \mathrm{O}_{3}$, and $\mathrm{MgO}_{\text {surface in }}$ contact with an hydrogen plasma. The interface is in all three cases perfectly absorbing as well as collisionless and the plasma is characterized by the parameters given in Table 1 